\title{
COMPOSICIÓN QUÍMICA Y CALIDAD INSTRUMENTAL DE CARNE DE BOVINO, LLAMA (Lama glama) Y CABALLO BAJO UN SISTEMA DE CRIANZA EXTENSIVA
}

\author{
Chemical Composition and Instrumental Quality of Bovine, Llama (Lama \\ glama) and Horse Meat Under an Extensive Production System
}

\author{
Lindon W. Mamani-Linares ${ }^{1,2,3}$, Carmen Gallo ${ }^{1}$
}

\section{Resumen}

\begin{abstract}
Se comparó la composición química y calidad instrumental de carne de bovino, llama, y caballo finalizados bajo pastoreo. Los bovinos $(n=31)$, llamas $(n=21)$ y caballos $(n=27)$ fueron faenados bajo procedimientos estándares de Chile. Las canales fueron refrigeradas durante 24 horas a $4{ }^{\circ} \mathrm{C}$. En el músculo Longissimus lumborum (LL) se determinó la composición proximal, contenido de colesterol y colágeno, $\mathrm{pH}$, color $\left(\mathrm{L}^{*}, \mathrm{a}^{*}, \mathrm{~b}^{*}, \mathrm{H}^{*}, \mathrm{C}^{*}\right)$, capacidad de retención de agua (WHC), pérdida de cocción, pérdida del descongelado y fuerza de corte Warner-Bratzler (WBSF). La carne de llama presentó mayores niveles de proteína y colágenos totales $(\mathrm{p}<0.05)$ y menor concentración de grasa y colesterol $(\mathrm{p}<0.05)$ en comparación a la carne de bovino y caballo. No se observaron diferencias estadísticas en porcentajes de humedad y cenizas entre las tres especies. Los valores de $\mathrm{L}^{*}$ (luminosidad) y $b^{*}$ (tenor de amarrillo) del LL de llama fueron mayores $(\mathrm{p}<0.05)$ a aquellos del bovino y caballo, mientras que los valores de a* (tenor de rojo) y $\mathrm{C}^{*}$ (croma) del LL de bovino fueron mayores $(\mathrm{p}<0.05)$. El LL bovino tuvo un $\mathrm{pH}$ más alto que el de la llama $(\mathrm{p}<0.05)$. Los porcentajes de expresión de jugosidad, pérdida total por cocción y por evaporación fueron similares, en tanto que el valor WBSF del caballo fue mayor $(\mathrm{p}<0.05)$.
\end{abstract}

Palabras clave : composición química, calidad de carne, bovino, llama, caballo

\section{Abstract}

This study compared the chemical composition and instrumental meat quality of bovine, llama and horse from animals finished under grazing systems. The bovines $(n=31)$, llamas $(n=21)$ and horses $(n=27)$ were slaughtered using Chilean standard procedures. Carcasses were stored for $24 \mathrm{~h}$ in a cold room $\left(4^{\circ} \mathrm{C}\right)$. The Longissimus lumborum muscle (LL) was collected for determining the chemical composition, cholesterol, collagen content, $\mathrm{pH}$, colour $\left(\mathrm{L}^{*}, \mathrm{a}^{*}, \mathrm{~b}^{*}, \mathrm{H}^{*}, \mathrm{C}^{*}\right)$, water holding capacity $(\mathrm{WHC})$ and Warner-Bratzler shear-force (WBSF). Llama meat had higher levels of protein and total collagen $(\mathrm{p}<0.05)$, and lower level of fat and cholesterol $(\mathrm{p}<0.05)$ than bovine and horse meat. No statistical

\footnotetext{
${ }^{1}$ Instituto de Ciencia Animal, ${ }^{2}$ Programa de Doctorado en Ciencias Veterinarias, Facultad de Ciencias Veterinarias, Universidad Austral de Chile, Chile

${ }^{3}$ E-mail: willymlmvzupea_2@hotmail.com
} 


\begin{abstract}
differences were found in moisture and ash percentages between species. The $\mathrm{L}^{*}$ (lightness) and $b^{*}$ (yellowness) values of llama LL were higher $(\mathrm{p}<0.05)$ than those in the bovine and horse, while the values of $\mathrm{a}^{*}$ (redness) and $\mathrm{C}^{*}$ (chroma) of LL bovine were greater $(\mathrm{p}<0.05)$. The $\mathrm{pH}$ in LL bovine meat was higher than in llama $(\mathrm{p}<0.05)$. WHC, total cooking loss and evaporation cooking loss were similar, while WBSF in horse was greater $(\mathrm{p}<0.05)$.
\end{abstract}

Key words : chemical composition, quality meat, bovine, llama, horse

\section{INTRODUCCIÓN}

La carne y productos cárnicos aportan valiosos nutrientes para la salud humana, proporcionando todos los aminoácidos esenciales (lisina, treonina, metionina, fenilalanina, triptófano, leucina, isoleucina y valina), asî como una buena cantidad de vitaminas, minerales y diversos micronutrientes esenciales para el crecimiento y desarrollo (NHMRC, 2006; Cabrera et al., 2009; McAfee et al., 2010); además, es una fuente importante de hierro, zinc y selenio, así como de vitaminas B6, B12 y D, y significativas cantidades de ácidos grasos esenciales como Omega-3 (n3) y ácido linoleico conjugado (CLA) (Ferguson, 2010; McAfee et al., 2010). Si se excluye la carne de la dieta humana y no se le reemplaza por otros alimentos similares, se puede afectar la salud por deficiencias de nutrientes (Chan, 2004).

Las propiedades sensoriales y las relacionadas con la salud y nutrición, se encuentran entre los factores de motivación más importantes para la compra de carne (Verbeke y Viaene 1999; Wood et al., 2003). Según Lawrie y Ledward (2006), los parámetros más importantes considerados en la evaluación de la calidad de la carne son apariencia, jugosidad, terneza y sabor. La carne debe tener un color atractivo que debe ser uniforme a lo largo de todo el corte.

Las propiedades tecnológicas de la carne permiten evaluar su aptitud y comportamiento en las etapas de conservación, comercialización, industrialización y prepara- ción para el consumo; dentro de ellas, el pH, la capacidad de retención de agua, la textura, el color y su estabilidad.

El pH es un parámetro importante relacionado con la susceptibilidad de la carne a su deterioro y se usa para decidir sobre el tipo de procesamiento al que se va a destinar la carne (Wirth, 1987). La capacidad de retención de agua (WHC) determina la pérdida de peso, principalmente por liberación de jugos, que se producen en toda la cadena de distribución y transformación de la carne, pudiendo también afectar la calidad de la carne, especialmente en términos de jugosidad y palatdbilided (Zhanget al., 2005). La terneza puede ser definida como la facilidad con que la carne se deja masticar; y está influenciada por la edad, sexo, peso y raza del animal, y estrés ante mortem (Muchenje et al., 2009). La terneza de la carne está en función del contenido de colágeno, la estabilidad térmica y la estructura de las miofibrillas del músculo (Monsón et al., 2005). El color está relacionado con la concentración de mioglobina y pigmentos proteicos presentes en el músculo, pudiendo ser afectado por las enzimas, la dieta, la edad del animal, e incluso la actividad realizada por el animal (Muchenje et al., 2009).

La escasez de estudios sobre algunos parámetros químicos y las características tecnológicas de carne de llamas y caballos motivaron este estudio. El objetivo de este trabajo fue determinar y comparar la composición química y la calidad instrumental de carne de bovino, llama y caballo. 


\section{Materiales y Métodos}

\section{Muestras}

Se utilizó el músculo Longissimus lumborum (LL) proveniente de 31 bovinos, 21 llamas y 27 caballos. Las muestras de bovinos fueron obtenidas de animales machos (cruzas con predominio de razas lecheras) de la región de Los Ríos, faenados en el matadero de Valdivia, y tipificadas en la categoría V (INN, 2002). Las muestras de llamas fueron obtenidas de animales de tipo Kh' ara, machos de 2 y 4 dientes permanentes, procedentes de la región de AricaParinacota, faenados en el matadero de Putre. Las muestras de caballos fueron adquiridas en carnicerías especializadas, seleccionadas al azar y visitadas en diferentes días, en la ciudad de Temuco, Chile. Los animales utilizados en el estudio procedieron de zonas con predominio de un sistema de producción extensivo, donde la alimentación está basada en praderas nativas o praderas cultivadas naturalizadas.

En el caso de bovinos y llamas, las canales fueron refrigeradas por 24 horas (0 - 4 $\left.{ }^{\circ} \mathrm{C}\right)$ previo a la toma de la muestra. Las muestras de las tres especies fueron transportadas en refrigeración al Laboratorio de Carnes del Instituto de Ciencia Animal, Facultad de Ciencias Veterinarias, Universidad Austral de Chile. Cada muestra de carne fue cortada en bifes de $2.5 \mathrm{~cm}$ de espesor, que fueron identificadas con códigos individuales y almacenadas a $-20{ }^{\circ} \mathrm{C}$. El análisis de las muestras se realizaron dentro de los 60 días de haberse adquirido las muestras de carne.

\section{Composición Química}

Un corte por muestra fue descongelado por 24 horas a $4{ }^{\circ} \mathrm{C} \mathrm{y}$, posteriormente, molido y homogenizado.

Composición proximal. Las muestras se analizaron en duplicado, utilizando los siguientes métodos: humedad, con el método oficial
AOAC 950.46, de secado al horno (AOAC, 1996); proteína bruta, con el método oficial AOAC 981.10, método Microkjeldahl Gerhardt que determina N en carne (AOAC, 1996); grasa, por el método de la norma chilena NCh 1370/3/1970; y cenizas con el método oficial AOAC 920.153, método directo de calcinación en mufla (AOAC, 1996).

Análisis de colesterol. Los lípidos de la muestra (10 g) se extrajeron con cloroformometanol (2:1), según metodología descrita por Folch et al. (1957). Una alícuota del extracto lipídico (3 ml) fue secada en nitrógeno y saponificada con $12 \% \mathrm{KOH}$ etanólico $(10 \mathrm{ml})$ durante 15 min a $80{ }^{\circ} \mathrm{C}$, de acuerdo a Bohac et al. (1988). Después de enfriar y añadir 5 $\mathrm{ml}$ de agua destilada, el material insaponificable se extrajo dos veces con 10 $\mathrm{ml}$ de hexano; $3 \mathrm{ml}$ del extracto fueron secados en nitrógeno, y redisueltos en $3 \mathrm{ml}$ de la fase móvil para cuantificar el colesterol por HPLC/UV (L-6200A Intelligent pump, L$4250 \mathrm{UV}$-VIS detector) equipado con una columna Phenomenex Luna 5 um C18 100 A ( $250 \times 4.60 \mathrm{~mm})$.

Contenido de colágeno. Las muestras molidas $(5 \mathrm{~g})$ se calentaron por $70 \mathrm{~min}$ a $77^{\circ} \mathrm{C}$ en solución de Ringer 1/4-strength y fueron divididas en fracciones de residuos y sobrenadante, siguiendo el procedimiento de Hill (1966). Cada fracción se hidroliza de forma individual en $6 \mathrm{~N} \mathrm{HCl}$ durante $6 \mathrm{~h}$ a $1 \mathrm{~atm}$ de presión y $102{ }^{\circ} \mathrm{C}$. Los niveles de hidroxiprolina se determinaron de acuerdo con Bergman y Loxley (1963). El contenido de colágeno (mg/g) se calculó a partir de los niveles de hidroxiprolina por los valores de conversión de 7.25 y 7.52 para el colágeno soluble e insoluble, respectivamente (Cross et al., 1973).

\section{Calidad Instrumental}

Tres cortes de cada muestra fueron descongelados a $4^{\circ} \mathrm{C}$ por 24 horas. Se calculó la pérdida de descongelado: (peso congelado - peso descongelado)/peso congelado, y el resultado se expresó en porcentaje. 
Color del músculo. El bife descongelado se removió de su envase y quedó expuesto al ambiente por una hora a $4{ }^{\circ} \mathrm{C}$. El color se midió sobre la superficie del corte con un espectrocolorímetro Miniscan XE Plus $\left(\right.$ Hunterlab $\left.^{\circledR}\right)$ con escala Lab y con una longitud de onda entre 400 y $700 \mathrm{~nm}$. Se determinó los valores de "L" (luminosidad), "a" (tenores de rojo-verde) y "b" (tenores de amarillo-azul).

$p H$. Se empleó un peachímetro con electrodo de penetración (Hanna ${ }^{\circledR}$, modelo HI 98230), introducido al centro del corte que fue empleado para medir color.

Capacidad de retención de agua o expresión de jugosidad (WHC, por sus siglas en inglés). Se determinó en uno de los bifes descongelados según el método de Grau y Hamm (1953) y modificado por Sañudo et al. (1986) (citado por Feed, 2009). Se tomó $20 \mathrm{~g}$ de muestra y se trituró durante $30 \mathrm{~s}$ con un martillo machacador. Se colocó $5 \mathrm{~g}$ entre dos papeles filtro y se sometieron a compresión entre dos placas de vidrio (dos cajas Petri) a presión de $2.250 \mathrm{~kg}$ durante $5 \mathrm{~min}$. Luego, se retiraron las placas y los papeles filtro, prestando atención de que no queden restos de carne pegados a los mismos, y se volvió a pesar la carne. La WHC se expresó en porcentaje.

Pérdida de cocción. Se emplearon los bifes utilizados para estimar el color y $\mathrm{pH}$. Se sometieron a cocción en un horno eléctrico a convección forzada (Trotter ${ }^{\circledR}$ ) a $170{ }^{\circ} \mathrm{C}$, hasta que la temperatura del centro de cada bife alcanzó los $70{ }^{\circ} \mathrm{C}$. La temperatura se determinó con termómetros digitales (Testo 925, Lenzkirch, Germany). La pérdida por cocción se calculó como la diferencia entre el peso de la muestra antes y después de la cocción, expresada como porcentaje del peso de la muestra inicial. Las pérdidas por evaporación (sustancias volátiles) fueron calculadas como diferencia entre la pérdida total de cocción y la pérdida por goteo (jugo de carne separada durante la cocción) (AMSA, 1995).
Textura (Warner-Bratzler shear force WBSF). Se midió utilizando el equipo WarnerBratzler (Salter ${ }^{\circledR}$ ), determinando la fuerza de cizalla, que corresponde a la fuerza $(\mathrm{kg})$ requerida para cortar un cilindro de carne cocida. Se utilizó el bife en que se determinó la pérdida por cocción. Las mediciones de fuerza de cizalla se realizaron en cilindros de carne extraídos de cada bife con un sacabocados de $1.2 \mathrm{~cm}$ de diámetro; el corte de cizalla se realizó al centro de cada cilindro (Beltrán y Roncales, 2000).

\section{Análisis Estadístico}

Los resultados fueron analizados a través del modelo lineal general (GLM) de Statistix para Windows v 8.0. Los promedios de la composición química y la calidad instrumental de la carne (LL) de bovinos, llamas y caballos fue comparado, y en casos de diferencia estadística se empleó la prueba de Tukey HSD, considerando $\mathrm{p}<0.05$ como diferencia estadística.

\section{Resultados y Discusión}

El porcentaje de proteína en el LL de llama fue más alto que en bovino y caballo $(p<0.05)$, mientras que el porcentaje de grasa fue más bajo ( $p<0.05$, Cuadro 1). En general, los resultados del LL de llama concuerdan con otros estudios (Cristofanelli et al., 2004; Salvá et al., 2009); sin embargo, se observa una discrepancia en el contenido de grasa intramuscular hallado por Polidori et al. (2007b) para Longissimus dorsi (LD) de llamas $(3.5 \%)$. Por otra parte, la composición proximal del LD de bovinos en el presente estudio fue similar a la descrita para bovinos jóvenes (Contreras, 2005; Göncü Karakok et al., 2010).

Las concentraciones de colesterol fueron diferentes en las tres especies $(\mathrm{p}<0.05)$, siendo más alta en caballos y más baja en llamas (Cuadro 1). En camélidos, Cristofanelli et al. (2004), encontraron valores de $51 \mathrm{mg} /$ 
Cuadro 1. Composición química (promedio \pm DE) del músculo Longissimus lumborum de bovino, llama y caballo

\begin{tabular}{lccc}
\hline Características & Bovino & Llama & Caballo \\
\hline Humedad (\%) & $73.72 \pm 0.84$ & $73.34 \pm 0.75$ & $72.41 \pm 1.51$ \\
Grasa (\%) & $2.27 \pm 0.10^{\mathrm{ab}}$ & $1.56 \pm 0.67^{\mathrm{b}}$ & $3.80 \pm 1.54^{\mathrm{a}}$ \\
Proteína (\%) & $22.46 \pm 0.61^{\mathrm{b}}$ & $23.88 \pm 0.77^{\mathrm{a}}$ & $21.41 \pm 1.31^{\mathrm{c}}$ \\
Ceniza (\%) & $1.19 \pm 0.02$ & $1.21 \pm 0.11$ & $1.25 \pm 0.08$ \\
Colesterol (mg/100g) & $49.85 \pm 1.34^{\mathrm{b}}$ & $39.04 \pm 1.92^{\mathrm{c}}$ & $66.8 \pm 2.34^{\mathrm{a}}$ \\
Colágeno total $(\mathrm{mg} / \mathrm{g})$ & $3.43 \pm 0.52^{\mathrm{c}}$ & $6.28 \pm 0.35^{\mathrm{a}}$ & $4.95 \pm 0.12^{\mathrm{b}}$ \\
Colágeno soluble $(\mathrm{mg} / \mathrm{g})$ & $0.82 \pm 0.14$ & $1.28 \pm 0.18$ & $0.60 \pm 0.01$ \\
Colágenos solubles $(\%)$ & $23.80 \pm 2.49^{\mathrm{a}}$ & $20.28 \pm 2.53^{\mathrm{b}}$ & $12.12 \pm 1.73^{\mathrm{c}}$ \\
\hline
\end{tabular}

a,b,c Superíndices diferentes dentro de cada parámetro indican diferencia

Cuadro 2. Calidad instrumental de músculo Longissimus lumborum de bovino, llama y caballo (promedio $\pm \mathrm{DE}$ )

\begin{tabular}{|c|c|c|c|c|}
\hline \multicolumn{2}{|l|}{ Características } & Bovino & Llama & Caballo \\
\hline \multicolumn{2}{|l|}{$\mathrm{pH}$} & $5.59 \pm 0.12^{\mathrm{a}}$ & $5.48 \pm 0.11^{\mathrm{b}}$ & $5.60 \pm 0.06^{\mathrm{a}}$ \\
\hline \multirow{5}{*}{ Color de músculo } & $\mathrm{L}^{*}$ & $25.04 \pm 2.28^{\mathrm{b}}$ & $34.92 \pm 2.77^{\mathrm{a}}$ & $21.93 \pm 2.77^{\mathrm{c}}$ \\
\hline & $a^{*}$ & $13.35 \pm 2.15^{\mathrm{a}}$ & $11.73 \pm 2.77^{\mathrm{b}}$ & $12.27 \pm 1.30^{\mathrm{ab}}$ \\
\hline & $b^{*}$ & $8.26 \pm 1.37^{\mathrm{b}}$ & $9.75 \pm 1.65^{\mathrm{a}}$ & $7.91 \pm 1.15^{\mathrm{b}}$ \\
\hline & $\mathrm{H}^{*}$ & $30.81 \pm 2.60^{\mathrm{c}}$ & $40.10 \pm 3.86^{\mathrm{a}}$ & $33.94 \pm 3.40^{\mathrm{b}}$ \\
\hline & $\mathrm{C}^{*}$ & $16.07 \pm 2.63^{\mathrm{a}}$ & $15.29 \pm 3.04^{\mathrm{ab}}$ & $14.81 \pm 1.34^{\mathrm{b}}$ \\
\hline \multicolumn{2}{|c|}{ Pérdidas por descongelación (\%) } & $5.77 \pm 1.34^{b}$ & $7.46 \pm 1.27^{\mathrm{a}}$ & $5.65 \pm 1.65^{\mathrm{b}}$ \\
\hline \multicolumn{2}{|c|}{ Expresión de jugosidad - WHC (\%) } & $16.88 \pm 2.74$ & $17.65 \pm 1.90$ & $16.55 \pm 2.38$ \\
\hline \multicolumn{2}{|c|}{ Pérdidas de cocción total $(\%)$} & $18.36 \pm 2.05$ & $17.52 \pm 1.96$ & $18.94 \pm 1.94$ \\
\hline \multicolumn{2}{|c|}{ Pérdidas por evaporación (\%) } & $15.91 \pm 1.69$ & $16.16 \pm 1.63$ & $16.45 \pm 1.68$ \\
\hline \multicolumn{2}{|c|}{ Pérdidas por goteo $(\%)$} & $2.44 \pm 0.75^{\mathrm{a}}$ & $1.37 \pm 0.56^{\mathrm{b}}$ & $2.50 \pm 0.95^{\mathrm{a}}$ \\
\hline \multicolumn{2}{|l|}{ WBSF $\left(\mathrm{kg} / \mathrm{cm}^{2}\right)$} & $1.84 \pm 0.57^{\mathrm{b}}$ & $2.16 \pm 0.40^{\mathrm{ab}}$ & $2.86 \pm 0.88^{\mathrm{a}}$ \\
\hline
\end{tabular}

a,b,c Superíndices diferentes dentro de cada parámetro indican diferencia estadística $(p<0.05)$

$L^{*}$ es una medición de luminosidad (valores más altos indican un color más claro)

$a^{*}$ es una medición de tenor de rojo (valores más altos indican un color más rojo)

$b^{*}$ es una medida de tenor de amarrillo (valor más altos indica un color más amarillo)

$\mathrm{C}^{*}($ Croma o Chroma $)=?\left(\mathrm{a}^{* 2}+\mathrm{b}^{\star 2}\right)$ (un valor más alto indica colores más vivos)

$\mathrm{H}^{*}($ Tono $)=\tan ^{-1}\left(\mathrm{~b}^{*} / \mathrm{a}^{*}\right)$ (un valor más alto indica un mayor cambio del eje de color rojo verdadero) 
$100 \mathrm{~g}$ en la alpaca y de $56 \mathrm{mg} / 100 \mathrm{~g}$ en la llama, en tanto que en llamas argentinas se reporta $52 \mathrm{mg} / 100 \mathrm{~g}$ en el músculo L. dorsi y $93 \mathrm{mg} / 100 \mathrm{~g}$ en la grasa renal (Coates y Ayerza, 2004). La literatura reporta rangos amplios de contenido de colesterol (27.5 a $57.27 \mathrm{mg} / 100 \mathrm{~g}$ ) en carne de bovinos (Duckett et al., 2009, Maggioni et al., 2010), y de ovinos y caprinos (65.88 a 67.88 y 56.55 a $64.64 \mathrm{mg} / 100 \mathrm{~g}$, respectivamente) (Costa $e t$ al., 2009; Madruga et al., 2009), mientras que se reporta valores altos $(64.2$ a $72.8 \mathrm{mg} / 100$ g) para el asno (Polidori et al., 2008). Por otro lado, Chizzolini et al. (1999) y Piironen et al. (2002), indican que la magnitud de variación en el contenido de colesterol de diferentes especies y músculos parece ser baja. En cierta medida, la alimentación puede influir el contenido de colesterol de la carne; así, Padre et al. (2007), señalan que los bajos niveles de colesterol en LD de bovinos finalizados en sistemas de pastoreo están relacionados con contenido de ácidos grasos no saturados de las pasturas.

El contenido de colágeno total en el LL fue mayor en la llama y menor en el bovino $(\mathrm{p}<0.05)$ (Cuadro 1), mientras que el porcentaje de colágenos solubles fue más bajo en el caballo ( $\mathrm{p}<0.05)$. En camélidos, Salva et al . (2009) reportaron $4.92 \mathrm{mg} / \mathrm{g}$ de colágeno total en carne de alpaca, valor inferior a los resultados del presente estudio en LL de llamas. En bovinos, los resultados de colágeno total $(3.43 \mathrm{mg} / \mathrm{g})$ fueron similares a los hallazgos en músculo Longissimus (2.35 - 4.52 $\mathrm{mg} / \mathrm{g}$ ) de toros jóvenes de 1 a 2 años de edad (Torrescano et al., 2003; Rhee et al., 2004; Serra et al., 2008), aunque mayores a los reportados en LD de corderos de 8 a 10 meses de edad $(2.6-2.9 \mathrm{mg} / \mathrm{g}$ ) (TschirhartHoelscher et al., 2006); sin embargo, en un estudio reciente, se reportan valores de 8.92 a $9.24 \mathrm{mg} / \mathrm{g}$ en el músculo LD de toros Nelore con 2 a 4 dientes permanentes y criados en pastura (Duarte et al., 2011). El colágeno es el tejido conectivo que más contribuye a la reducción de la terneza de la carne. Aunque el colágeno constituye menos del $2 \%$ del músculo esquelético, está asociado con la dureza y puede ser muy resistente a la degradación física durante la cocción (Weston et al. 2002).

No se encontraron diferencias significativas entre especies en porcentajes de humedad y ceniza (Cuadro 1).

Los resultados de los parámetros de calidad tecnológica de la carne de bovino, llama y caballo se muestran en el Cuadro 2. El $\mathrm{pH}$ en los bifes de llamas fue menor que el de los bovinos y caballos $(\mathrm{p}<0.05)$, pero se encuentra dentro de los rangos normales. En camélidos, Salvá et al. (2009) reportaron un $\mathrm{pH}$ de 5.63 en carne de alpaca, mientras que Cristofanelli et al. (2004) indicaron valores a las 24 horas de la faena de 5.60 y 5.57 en llamas y alpacas, respectivamente. El pH es un parámetro relacionado con el nivel de deterioro de la carne y se usa para decidir sobre el destino de la carne (Wirth, 1987), y depende de factores tales como el estrés ante mortem, factores genéticos predisponentes a dicho estrés, condiciones post mortem, sexo, clase o categoría de animal, tipo de alimentación recibida en la finalización, y región anatómica en que se mide, entre otros (Tarrant y Grandin 1993; Apaoblaza et al., 2008; Herrera, 2008).

Cuando el animal se somete a estrés prolongado y consume prácticamente sus reservas de glucógeno, no hay glicólisis anaerobia post mortem, por lo que las carnes obtenidas presentan la condición conocida como "corte oscuro" (Hood y Tarrant 1980). Los camélidos parecen ser poco susceptibles a la pérdida de calidad de la carne debida al estrés, de allí que suelen presentar $\mathrm{pH}$ altos; por otro lado, el proceso de glicólisis en caballos y asnos es similar a otras carne rojas (Polidori et al., 2008; Tateo et al., 2008).

No se encontró diferencias estadísticas entre especies en términos de WHC. Los resultados de expresión de jugosidad de la llama fueron menores a otros reportes en carne de alpaca (26.41\%; Salvá et al., 2009) y en carne de alpaca y llama (Cristofanelli et 
al., 2004). En este último, empleando el método de imbibición de jugo sobre papel de filtro colocado en la superficie muscular del Longissimus thoracis y lumborum, encontraron que la WHC de la carne de camélidos no era muy diferente a la carne de otras especies.

Las pérdidas por cocción total y por evaporación en la carne de las tres especies fueron similares; sin embargo, la carne de llama presentó la menor pérdida por goteo $(\mathrm{p}<0.05)$. La pérdida de cocción en la carne de llama fue más baja que en otros estudios con carne de alpaca (Salvá et al., 2009) y de novillos (Duckett et al., 2007), pero similar a otros reportes para carne de corderos y chivos (Lee et al., 2008). Por otro lado, los resultados de pérdida de cocción de LL de carne de caballo están entre los valores reportados (15.48 a 26.57\%) (Tateo et al., 2008; Franco et al., 2011). La expresión de jugosidad se encuentra afectada por el contenido de grasa intramuscular (infiltración o marmoleo), por lo cual la carne magra, especialmente de animales jóvenes, puede presentar carnes menos jugosas.

Los valores de Warner-Bratzler shear force (WBSF) o fuerza de corte fueron menores en el bovino en comparación la carne del caballo ( $>>0.05$, Cuadro 2). En trabajos realizados con carne (Longissimus dorsi) de alpaca se registran valores más altos (4.67 $6.06 \mathrm{~kg} / \mathrm{cm}^{2}$ ) (Polidori et al., 2007a; Salvá et al., 2009); en cambio, los valores de WBSF encontrados para el bovino se encuentran dentro del rango reportado para novillos finalizados a pastoreo con 1.9 a $2.88 \mathrm{~kg} / \mathrm{cm}^{2}$ (Contreras, 2005; Monsón et al, 2005; Koger et al., 2010), y para caballos (2.53 a $5.98 \mathrm{~kg} /$ $\mathrm{cm}^{2}$ ) (Tateo et al., 2008; Franco et al., 2011). La WBSF es un indicador de la terneza, que es definida como la facilidad con que la carne se deja masticar. Está influenciada por la edad, sexo, peso y raza del animal, y estrés ante mortem (Muchenje et al., 2009). Altos valores de WBSF están relacionados con alta actividad de calpastatina, baja grasa intramus- cular, baja de fragmentación miofibrilar, altos niveles de colágeno totales y bajo colágeno soluble o la combinación de estos factores (Kannan et al., 2006; Nakamura et al., 2010; Duarte et al., 2011).

En relación al color de la carne, el valor de croma $\left(\mathrm{C}^{*}\right)$ fue más alto en el bovino y más bajo en el caballo, lo que significa que el LL de bovino presenta colores más vivos y el del caballo colores más apagados. Los resultados de $\mathrm{L}^{*}$ en la llama fueron similares a los de alpaca, pero diferentes en $\mathrm{a}^{*} \mathrm{y} \mathrm{b}^{*}$ a los de Salvá et al. (2009), lo que significa un rojo más claro, que puede ser debido al mayor contenido de grasa intramuscular $(2.05 \%)$ y al suplemento de grano recibido por las alpacas de dicho estudio.

Los valores de $a^{*}$ y $b^{*}$ encontrados para la carne de bovino están dentro de rangos reportados para bovinos criados a pastoreo, aunque con valores más bajos de luminosidad (Realini et al., 2004; Orellana et al., 2009; Muchenje et al., 2009); en tanto que para el músculo Longissimus de caballos y asnos se reportan valores entre 28.4 a 36.7 para L*, de 10.43 a 24.2 para a* y de -1.67 a 9.6 para b* (Tateo et al., 2008; Polidori et al., 2009; Juarez et al., 2009). La intensidad de b* se ve influida por el contenido y el color de la grasa intramuscular presente en la carne. En animales de mayor edad se encuentra una mayor concentración de caroteno, especialmente en animales al pastoreo (Priolo et al., 2002).

\section{Conclusiones}

? La carne de llama tiene alto contenido de proteína y bajo contenido de grasa y colesterol comparado a la carne del bovino y del caballo.

? La carne de llama puede compararse favorablemente en color, apariencia y textura con la carne del bovino, en tanto que la carne del caballo presenta color rojo oscuro y opaco. 


\section{Literatura Citada}

1. [AMSA] American Meat Science Association. 1995. Research guidelines for cookery and sensory evaluation and instrumental tenderness measurement of fresh meat. Chicago, IL: AMSA.p 96-101.

2. AOAC. 1996. Official methods of analysis. Washington, DC: Association of Official Analytical Chemists. p 143-160.

3. Apaoblaza A, Pulido R, Gallo C. 2008. Efecto de una suplementación en base a maíz roleado, sobre la prevención de corte oscuro (CO) y algunas variables de calidad de la carne en novillos. En: Libro de Resúmenes XXXIII Congreso Anual de la Sociedad Chilena de Producción Animal. Valdivia, Chile. p 251-252.

4. Beltrán JA., Roncalés P. 2000. Determinación de la textura. In: Cañeque V, Sañudo C (eds). Metodología para el estudio de la calidad de la canal y de la carne en rumiantes. Madrid: Instituto Nacional de Investigación y Tecnología Agraria y Alimentaria. p 168-174.

5. Bergman I, Loxley R. 1963. Two improved and simplified methods for the spectrophotometric determination of hydroxyoriline. Anal Chem 35: 19611965.

6. Bohac CE, Rhee KS, Cross HR, Ono

K. 1988. Assessment of methodologies for colorimetric cholesterol assay of meats. J Food Sci 53: 1642-1644.

7. Cabrera E, Varela JJ, Campos CA, Castillo A. 2009. Inocuidad de carnes rojas. En: Bianchi G, Feed O (eds). Introducción a la ciencia de la carne. Buenos Aires, Argentina: Hemisferio sur. $\mathrm{p}$ 129-179.

8. Chan W. 2004. Macronutrients in meat. In: Jensen WK, Devine C, Dikeman M (eds). Encyclopaedia of meat science. Oxford: Elsevier Academic Press. p 614618.

9. Chizzolini R, Zanardi E, Dorigoni V, Ghidini S. 1999. Calorific value and cholesterol content of normal and lowfat meat and meat products. Trends Food Sci Tech 10: 119-128.
10. Coates W, Ayerza R. 2004. Fatty acid composition of llama muscle and internal fat in two Argentinian herds. Small Rum Res 52: 231-238.

11. Contreras SA. 2005. Efecto de la suplementación con cereales y afrecho de soya en la engorda de novillos sobre las características de canal y calidad de carne. Tesis de Médico Veterinario. Escuela de Medicina Veterinaria, Universidad Austral de Chile, Valdivia, Chile. 67 p.

12. Costa RG, Malveira AS, de Azevedo PS, Ramos do Egypto RC, Madruga MS, de Araújo JT. 2009. Lipid profile of lamb meat from different genotypes submitted to diets with different energy levels. R Bras Zootec 38: 532-538.

13. Cristofanelli S, Antonini M, Torres D, Polidori P, Renieri C. 2004. Meat and carcass quality from Peruvian llama (Lama glama) and alpaca (Lama pacos). Meat Sci 66: 589-593.

14. Cross HR, Carpenter ZL, Smith GC. 1973. Effect of intramuscular collagen and elastin on bovine muscle tenderness. J Food Sci 38: 998-1003.

15. Duarte MS, Paulino PVR, Fonseca MA, Diniz LL, Cavali J, Serao NVL, Gomide LAM, et al. 2011. Influence of dental carcass maturity on carcass traits and meat quality of Nellore bulls. Meat Sci 88: 441-446.

16. Duckett SK, Neel JPS, Sonon Jr RN, Fontenot JP, Clapham WM, Scaglia G. 2007. Effects of winter stocker growth rate and finishing system on: II. Ninth tenth eleventh-rib composition, muscle color and palatability. J Anim Sci 85: 2691-2698.

17. Duckett SK, Neel JPS, Fontenot JP, Clapham WM. 2009. Effects of winter stocker growth rate and finishing system on: III. Tissue proximate, fatty acid, vitamin, and cholesterol content. J Anim Sci 87: 2961-2970.

18. Feed O. 2009. Metodología para la evaluación de las características cualitativas de la canal y de la carne. En: Bianchi G, Feed O (eds). Introducción a la ciencia de la carne. Buenos Aires, Argentina: Hemisferio Sur. p 181-239. 
19. Ferguson LR. 2010. Meat and cancer. Meat Sci 84: 308-313.

20. Folch J, Lees M, Stanley GHSA. 1957. Simple method for the isolation and purification of total lipides from animal tissues. J Biol Chem 226: 497-509.

21. Franco D, Rodríguez E, Purriños L, Crecente S, Bermúdez R, Lorenzo JM. 2011. Meat quality of "Galician Mountain" foals breed. Effect of sex, slaughter age and livestock production system. Meat Sci 88: 292-298.

22. Grau R, Hamm R. 1957. Über das wasserbindungsvermögen des säugetiermuskels II Mitt. Über die bestimmung der wasserbindung des muskels. Z Lebensm Unters For 105: 446-460.

23. Göncü Karakök S, Ozogul Y, Saler M, Ozogul F. 2010. Proximate analysis. Fatty acid profiles and mineral contents of meats: A comparative study. J Muscle Foods 21: 210-223.

24. Herrera CA. 2008. Análisis descriptivo de factores asociados a la presentación de contusiones y $\mathrm{pH}$ elevado en canales de bovinos de distintas procedencias geográficas. Tesis de Médico Veterinario. Escuela de Medicina Veterinaria, Universidad Austral de Chile, Valdivia, Chile. $41 \mathrm{p}$.

25. Hill F. 1966. The solubility of intramuscular collagen in meat animals of various ages. J Food Sci 31: 161-166.

26. Hood DE, Tarrant PV. 1980. The problem of dark-cutting in beef. The Hague: Martinus Nijhoff. 501 p.

27. [INN] Instituto Nacional de Normalización, Chile. 2002. Canales de Bovino - Definiciones y tipificación. Norma Chilena Oficial NCH. 1306. Of. 93.

28. Juárez M, Polvillo O, Gómez MD, Alcalde MJ, Romero F, Varela M. 2009. Breed effect on carcass and meat quality of foals slaughtered at 24 months of age. Meat Sci 83: 224-228.

29. Kannan G, Gadiyaram KM, Galipalli S, Carmichael A, Kouakou B, Pringle $T D$, et al. 2006. Meat quality in goats as influenced by dietary protein and energy levels and postmortem aging. Small Rumin Res 61: 45-52.
30. Koger TJ, Wulf DM, Weaver AD, Wright CL, Tjardes KE, Mateo KS, Engle TE, et al. 2010. Influence of feeding various quantities of wet and dry distillers grains to finishing steers on carcass characteristics, meat quality, retail-case life of ground beef, and fatty acid profile of longissimus muscle. $\mathbf{J}$ Anim Sci 88: 3399-3408.

31. Lawrie RA, Ledward DA. 2006. Lawrie's meat science. $7^{\text {th }}$ ed. Cambridge, England: Woodhead Publishing. $442 \mathrm{p}$.

32. Lee JH, Kannan G, Eega KR, Kouakou B, Getz WR. 2008. Nutritional and quality characteristics of meat from goats and lambs finished under identical dietary regime. Small Rumin Res 74: 255-259.

33. McAfee AJ, McSorley EM, Cuskelly GJ, Moss BW, Wallace JMW, Bonham MP, Fearon AM. 2010. Red meat consumption: An overview of the risks and benefits. Meat Sci 84: 1-13.

34. Madruga MS, Medeiros EJL, Sousa WH, Cunha MGG, Filho JMP, Queiroga RCRE. 2009. Chemical composition and fat profile of meat from crossbred goats reared under feedlot systems. R Bras Zootec 38: 547-552.

35. Maggioni D, de Araújo Marques J, Rotta PP, Perotto D, Ducatti T, Visentainer JV, Nunes do Prado I. 2010. Animal performance and meat quality of crossbred young bulls. Livestock Sci 127: 176-182.

36. Monsón F, Sañudo C, Sierra I. 2005. Influence of breed and ageing time on the sensory meat quality and consumer acceptability in intensively reared beef. Meat Sci 71: 471-479.

37. Muchenje V, Dzama K, Chimonyo M, Strydom P, Hugo A, Raats J. 2009. Some biochemical aspects pertaining to beef eating quality and consumer health: A review. Food Chem 112: 279-289.

38. Nakamura YN, Tsuneishi E, Kamiya M, Yamada A. 2010. Histological contribution of collagen architecture to beef toughness. J Food Sci 75: 73-77. 
39. [NHMRC] National Health and Medical Research Council. 2006. Nutrient reference values for Australia and New Zealand. Canberra: Department of Health and Ageing, NHMRC. 317 p.

40. Orellana C, Peña F, García A, Perez $J$, Martos J, Domenech V, Acero R. 2009. Carcass characteristics, fatty acid composition, and meat quality of Criollo Argentino and Braford steers raised on forage in a semi-tropical region of Argentina. Meat Sci 81: 57-64.

41. Padre RG, Aricetti JA, Gomes STM, Goes RHTB, Moreira FB, Prado IN, Visentainer JV, et al. 2007. Analysis of fatty acids in Longissimus muscle of steers of different genetic breeds finished in pasture systems. Livestock Sci 110: 57-63.

42. Piironen V, Toivo J, Lampi AM. 2002. New data for cholesterol contents in meat, fish, milk, eggs and their products consumed in Finland. J Food Composition Anal 15: 705-713.

43. Polidori P, Antonini M, Torres D, Beghelli D, Renieri C. 2007a. Tenderness evaluation and mineral levels of llama ( Lama glama) and alpaca (Lama pacos) meat. Meat Sci 77: 599-601.

44. Polidori P, Renieri C, Antonini M, Passamonti P, Pucciarelli F. 2007b. Meat fatty acid composition of llama (Lama glama) reared in the Andean highlands. Meat Sci 75: 356-358.

45. Polidori P, Cavallucci C, Beghelli D, Vincenzetti S. 2009. Physical and chemical characteristics of donkey meat from Matina Franca breed. Meat Sci 82: 469-471.

46. Polidori P, Vincenzetti S, Cavallucci C, Beghelli D. 2008. Quality of donkey meat and carcass characteristics. Meat Sci 80: 1222-1224.

47. Priolo A, Micol D, Agabriel J, Prache $S$, Dransfield E. 2002. Effect of grass or concentrate feeding systems on lamb carcass and meat quality. Meat Sci 62: 179-185.
48. Realini CE, Duckett SK, Brito GW, Dalla Rizza M, De Mattos D. 2004. Effect of pasture vs. concentrate feeding with or without antioxidants on carcass characteristics, fatty acid composition, and quality of Uruguayan beef. Meat Sci 66: 567-577.

49. Rhee MS, Wheeler TL, Shackelford $S D$, Koohmaraie M. 2004. Variation in palatability and biochemical traits within and among eleven beef muscles. J Anim Sci 82: 534-550.

50. Salvá BK, Zumalacárregui JM, Figueira AC, Osorio MT, Mateo J. 2009. Nutrient composition and technological quality of meat from alpacas reared in Peru. Meat Sci 82: 450455.

51. Serra X, Guerrero L, Guardia M, Gil M, Sañudo C, Panea B, et al. 2008. Eating quality of young bulls from three Spanish beef breed-production systems and its relationships with chemical and instrumental meat quality. Meat Sci 79: 98-104.

52. Tarrant PV, Grandin T. 1993. Cattle transport. In: Livestock handling and transport. United Kingdom: CAB Int. $p$ 109-126.

53. Tateo A, De Palo $P$, Ceci $E$, Centoducati P. 2008. Physicochemical properties of meat of Italian Heavy Draft horses slaughtered at the age of eleven months. J Anim Sci 86: 1205-1214.

54. Torrescano G, Sánchez-Escalante A, Giménez B, Roncalés P, Beltrán J. 2003. Shear values of raw samples of 14 bovine muscles and their relation to muscle collagen characteristics. Meat Sci 64: 85-91.

55. Tschirhart-Hoelscher TE, Baird BE, King DA, McKenna DR, Savell JW. 2006. Physical, chemical, and histological characteristics of 18 lamb muscles. Meat Sci 73: 48-54.

56. Verbeke W, Viaene J. 1999. Beliefs, attitude and behaviour towards fresh meat consumption in Belgium: Empirical evidence from a consumer survey. Food Qual Prefer 10: 437-445. 
57. Weston AR, Rogers RW, Althen TG. 2002. Review: The role of collagen in meat tenderness. Prof Anim Sci 18: 107-111.

58. Wirth F. 1987. Tecnología para la transformación de carne de calidad anormal. Fleischwirtschaft español 1: 22-28.

59. Wood JD, Richardson RI, Nute GR,
Fisher AV, Campo MM, Kasapidou E. 2003. Effects of fatty acids on meat quality: A review. Meat Sci 66: 21-32.

60. Zhang SX, Farouk MM, Young OA, Wieliczko KJ, Podmore C. 2005. Functional stability of frozen normal and high pH beef. Meat Sci 69: 765-772. 